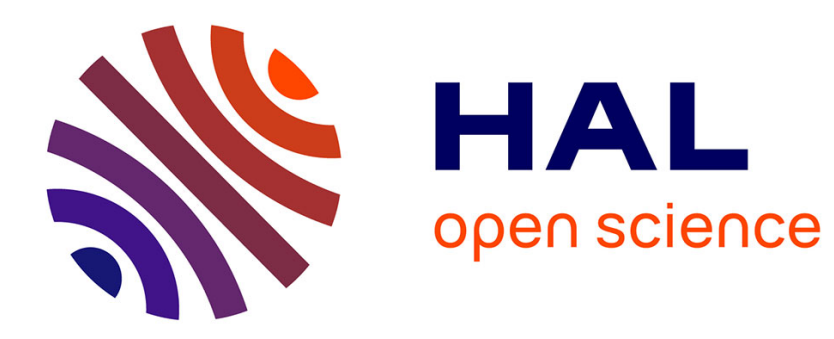

\title{
Réflexion autour de la question de l'évaluation du handicap psychique
}

\author{
B. Falissard
}

\section{To cite this version:}

B. Falissard. Réflexion autour de la question de l'évaluation du handicap psychique. Annales MédicoPsychologiques, Revue Psychiatrique, 2010, 168 (10), pp.768. 10.1016/j.amp.2010.09.019 . hal00698861

\section{HAL Id: hal-00698861 https://hal.science/hal-00698861}

Submitted on 18 May 2012

HAL is a multi-disciplinary open access archive for the deposit and dissemination of scientific research documents, whether they are published or not. The documents may come from teaching and research institutions in France or abroad, or from public or private research centers.
L'archive ouverte pluridisciplinaire HAL, est destinée au dépôt et à la diffusion de documents scientifiques de niveau recherche, publiés ou non, émanant des établissements d'enseignement et de recherche français ou étrangers, des laboratoires publics ou privés. 


\section{Accepted Manuscript}

Title: Réflexion autour de la question de l'évaluation du handicap psychique

Author: B. Falissard

PII: $\quad$ S0003-4487(10)00332-X

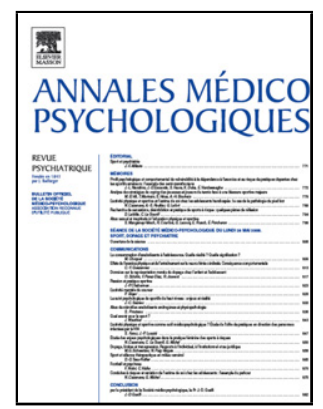

DOI: doi:10.1016/j.amp.2010.09.019

Reference: AMEPSY 1259

To appear in: Annales Médico-Psychologiques

Please cite this article as: Falissard B, Réflexion autour de la question de l'évaluation du handicap psychique, Annales medio-psychologiques (2010), doi:10.1016/j.amp.2010.09.019

This is a PDF file of an unedited manuscript that has been accepted for publication. As a service to our customers we are providing this early version of the manuscript. The manuscript will undergo copyediting, typesetting, and review of the resulting proof before it is published in its final form. Please note that during the production process errors may be discovered which could affect the content, and all legal disclaimers that apply to the journal pertain. 
Communication

\title{
Réflexion autour de la question de l'évaluation du handicap psychique Reflection on the question of the evaluation of the psychic handicap B. Falissard
}

INSERM U669, Maison de Solenn, 97, Boulevard de Port-Royal, 75679 Paris cedex 14, France

Auteur correspondant : Pr Bruno Falissard, INSERM U669, Maison de Solenn, 97, Boulevard de Port-Royal, 75679 Paris cedex 14, France

Tél. : 0158412851

Adresse email : falissard_b@wanadoo.fr

\section{Résumé}

Ce texte constitue le résumé de la communication présentée par le Pr Bruno Falissard.

Mots clés : Handicap psychique ; Prise en charge ; Santé mentale

\begin{abstract}
This text constitutes the summary of the presentation submitted by Pr Bruno Falissard.
\end{abstract}

Keywords: Care; Mental health; Psychic handicap

Pour Bruno Falissard, une évaluation (étymologiquement : donner une valeur) repose non seulement sur une arrière-pensée morale (la valeur) mais, de façon plus technique, sur la question de la mesure. D’où la question: comment mesurer, comment représenter numériquement, de façon simple, une caractéristique comme le handicap psychique ?

Pour lui, la mesure de la santé mentale relève de deux paradigmes ou modèles :

- l’un qui relève de la phénoménologie, c’est-à-dire de l'appréciation globale et intuitive de certaines caractéristiques. Par exemple, on peut mesurer assez simplement le niveau de tristesse de quelqu'un avec qui l'on discute en attribuant un nombre de un à dix, 
comme on le fait pour mesurer un niveau de douleur avec une échelle visuelle analogique. Cela est possible parce que la notion de tristesse est élémentaire ;

- l'autre paradigme doit être utilisé lorsque le concept est trop compliqué (et c'est le cas du handicap psychique). Il faut alors le diviser en plusieurs éléments que l'on mesurera d'abord un à un pour les assembler ensuite en une seule mesure. Cela est possible à condition de définir de façon explicite et consensuelle le phénomène que l'on mesure, et que les éléments isolés puissent être regroupés, additionnés, de manière à ce que l’ensemble donne un résultat significatif.

Pour illustrer le passage du plus simple au plus compliqué, Bruno Falissard a repris l'exemple de la mesure de la tristesse (élémentaire), et celui de la dépression (plus complexe). Les instruments de mesure habituels obligent à « atomiser » la dépression, à la décomposer en éléments isolés, c’est-à-dire en une somme de symptômes caractéristiques de cette maladie. C’est la mesure de chacun des symptômes qui permet d’obtenir une échelle de dépression relativement consensuelle.

Pour se rapprocher un peu plus du handicap psychique, il est utile d'aborder dans un premier temps, selon lui, la question du «fonctionnement ». L'auteur rappelle à ce propos la définition du fonctionnement donnée dans le dictionnaire de l'Académie: «Niveau de réalisation des activités, obligations et devoirs inhérents à notre existence. » Ce qui revient à dire, souligne-t-il, que « fonctionner, c'est réaliser des choses, et en particulier ses devoirs »; une définition qu’il met en regard d’une autre définition du fonctionnement : «Rôle joué par un individu dans la société. »

Ces définitions entraînent donc un certain nombre de questionnements : quels sont les obligations et devoirs d'un sujet dans son existence? Comment les mesurer, et plus généralement, comment mesurer le niveau d’aspirations et de réalisations ? Certes, en psychopathologie - indépendamment des mesures du handicap -, on utilise des échelles de fonctionnement : si on a un travail, des enfants, si on fait du sport, de la musique... alors on fonctionne. De là cette remarque, « On voit bien que ce ne sont pas de très bons instruments. Ils reposent sur des hypothèses discutables basées sur l'appréciation de ce qu'un individu doit faire de son existence... », ce qui laisse donc place aux débats philosophiques.

Les mêmes questions philosophiques se posent quant à l'estimation de la qualité de vie. La définition de l'OMS, «perception de sa position dans la vie, dans le contexte d'une culture en rapport avec ses attentes », amène à se demander : sur quoi repose l'évaluation d'une qualité de vie ? Cela fait 2500 ans que les philosophes essaient de réfléchir à la question du «souverain bien », en d'autres termes de ce qui fait la qualité de vie. «Être 
universitaire et avoir l'honneur de parler devant vous, comme moi ? Satisfaire une ambition, un narcissisme un peu basique, ou le contraire, comme pensent certains philosophes ? », interroge Bruno Falissard.

Et l'on arrive au concept de handicap proprement dit : « ce sont les aspects négatifs de l'interaction entre l'individu et son contexte » selon l'OMS. Encore une fois, Bruno Falissard se demande : en quoi consistent ces interactions ? Ici aussi, cela pose la question de la place de l’homme dans la vie, dans la société, dit-il. Et cela conduit à réfléchir à la manière de mieux traiter les patients, de sortir de notre conditionnement, de la triade - symptômes, diagnostic, traitement - à laquelle on ajoute l'observation des symptômes résiduels, et voir comment il est possible d'améliorer les choses. Pourquoi ne pas demander au sujet lui-même ce qu'il veut réaliser dans sa vie, ce que les proches et lui voudraient organiser ? Mais le contexte dont parle l’OMS, autrement dit la société dans laquelle vit l'individu, peut-il permettre à celui-ci de vivre comme il en a envie ? Cela sans provoquer de tensions... L'exemple extrême du marquis de Sade au XXVIII ${ }^{\mathrm{e}}$ siècle illustre les difficultés à y répondre. On peut considérer, d’une certaine façon, que la société de l'époque ne lui a pas permis de s’épanouir comme il l'aurait voulu. Il a été emprisonné et a fini sa vie à Esquirol. On a bien la démonstration d'une interaction négative d'un individu et de son contexte. Le marquis était-il pour autant un handicapé psychique ?

Bruno Falissard fait également observer que l'on retrouve la notion de justice - ou plutôt d'injustice - derrière le mot handicap, en rappelant son étymologie, hand in cap (main dans le chapeau) : en Angleterre, les possédants, ceux qui avaient plusieurs enfants, n’avaient pas la même probabilité d’être tirés au sort (dans le chapeau) pour partir sous les drapeaux.

Ces remarques ont pour objet de montrer que le handicap est toujours révélateur d'une tension entre plusieurs modèles pour décider de la prise en charge d'une personne, notamment dans le cadre d'une maladie chronique, et, d'autre part, de souligner qu'une idée de compensation est toujours sous-tendue.

Avec l'évaluation du handicap, il y a débat sur ce que devrait être la prise en charge de nos semblables qui sont en difficulté et qui risquent d’y rester un certain temps. Que devonsnous compenser ? Comment ? Jusqu'où ? Qu'est-ce que la société est prête à accepter en termes de compensation?

\section{Conflit d'intérêt : aucun}

